\title{
Hemodynamic Analysis of Postoperative Rupture of Unruptured Intracranial Aneurysms after Placement of Flow-Diverting Stents: A Matched Case-Control Study
}

\author{
(DW. Li, (D) Z. Tian, (DW. Zhu, (D).S. Zhang, (D). Wang, (D). Zhang, (D). Wang, (D) X. Yang, and (D). Liu
}

\section{ABSTRACT}

BACKGROUND AND PURPOSE: Postoperative rupture of intracranial aneurysm has been reported as a fatal complication after flow-diverter placement. We assessed several hemodynamic variables to explore risk factors in the postoperative rupture process.

MATERIALS AND METHODS: We enrolled 10 patients with intracranial aneurysms, treated with flow diverters between September 2014 and December 2018, who experienced postoperative aneurysm rupture (postoperative aneurysm rupture group). We matched these subjects 1:2 with 20 patients with postoperative unruptured (postoperative unruptured group) intracranial aneurysms based on clinical and morphologic factors. Using computational fluid dynamics, we assessed hemodynamic changes pre- and posttreatment between the 2 groups on a number of qualitative and quantitative parameters.

RESULTS: In the postoperative aneurysm rupture group, the proportion of patients with aneurysms with an unstable flow pattern increased to $60.0 \%$ after treatment, while the proportion in the postoperative unruptured group decreased to $20.0 \%$, a significant difference between the 2 groups $(P=.028)$. Energy loss in the postoperative unruptured group decreased after treatment but increased in the postoperative aneurysm rupture group. The reduction ratio of energy loss showed a significant difference between the 2 groups $(22.73 \% \pm 53.59 \%$ for postoperative unruptured versus $-158.81 \% \pm 183.95 \%$ for postoperative aneurysm rupture, $P=.02$ ). Other parameters and changes of pre- and posttreatment hemodynamic parameters showed no significant difference between 2 groups.

CONCLUSIONS: Compared with pretreatment, unstable flow pattern and higher energy loss after Pipeline Embolization Device placement for intracranial aneurysm may be the important hemodynamic risk factors related to delayed aneurysm rupture.

ABBREVIATIONS: CFD = computational fluid dynamics; EL = energy loss; IA = intracranial aneurysm; LSA = low wall shear stress area; OG = occlusion grade; OSI = oscillatory shear index; POR = postoperative ruptured; POU = postoperative unruptured; RRT = relative residual time; TAWSS = time-averaged wall shear stress; WSS $=$ wall shear stress

E ndoluminal reconstruction with the Pipeline Embolization Device (PED; Covidien, Irvine, California) has been widely used in large and giant intracranial aneurysms (IAs). The complete occlusion rate reported in the literature is encouraging; ${ }^{1-4}$ however, postoperative delayed rupture of IAs has been reported as a fatal complication. ${ }^{5-8}$ A meta-analysis showed that the incidence of subarachnoid hemorrhage after flow-diverter placement was $4.0 \%$, with a higher incidence in patients with large or giant

Received June 5, 2019; accepted after revision August 23.

From the Department of Interventional Neuroradiology (W.L., Z.T., W.Z., Y.S.Z., K.W., Y.Z., X.Y., J.L.), Beijing Neurosurgical Institute and Beijing Tiantan Hospital, Capital Medical University, Beijing, China; and Department of Neurosurgery (Y.W.), The First Affiliated Hospital of Nanchang University, Nanchang University, Nanchang, China.

W. Li and Z. Tian contributed equally to this work.

This work was supported by the National Key Research and Development Plan of China (grant No. 2016YFC1300800), the National Natural Science Foundation of China (grant Nos. 81220108007, 81801156, 81801158, 81471167, and 81671139), and the Special Research Project for Capital Health Development (grant No. 2018-4-1077).
IAs. ${ }^{9}$ The potential mechanisms of postoperative delayed rupture are still unclear, however.

Treatment of IAs with flow diverters and coils is thought to promote thrombosis and treat the aneurysm. Another hypothesis is that thrombosis and inflammation make the aneurysm wall more vulnerable. ${ }^{7,10,11}$ With flow diverters and coils designed to cure the aneurysm by modifying the flow, hemodynamic changes are also thought to play an important role in the postoperative rupture process. However, hemodynamic research of

Please address correspondence to Jian Liu, MD, Department of Interventional Neuroradiology, Beijing Neurosurgical Institute and Beijing Tiantan Hospital, Capital Medical University, NansanhuanXilu 119, Fengtai District, Beijing, China, 100070; e-mail: jianliu_ns@163.com

\footnotetext{
- Indicates open access to non-subscribers at www.ajnr.org

$\equiv$ Indicates article with supplemental on-line tables.

Indicates article with supplemental on-line photos.

http://dx.doi.org/10.3174/ajnr.A6256
} 
postoperative rupture is limited. ${ }^{5,12}$ In this study, we used the computational fluid dynamics (CFD) method and advanced virtual stent placement technique to explore the hemodynamic factors associated with postoperative delayed rupture. We compared unruptured IAs that ruptured after PED treatment with matched aneurysms that did not rupture during the observation period. We investigated whether hemodynamic parameters could predict postoperative aneurysm rupture.

\section{MATERIALS AND METHODS Patient Selection}

This retrospective, matched case-control study was approved by the ethics committee of Beijing Tiantan hospital. We reviewed the medical records and image data in our aneurysm data base of patients diagnosed with unruptured IAs between September 2014 and December 2018. We identified 388 patients with 426 unruptured IAs treated with PEDs. From the CT scans, we identified patients who had a delayed aneurysmal subarachnoid hemorrhage after PED placement; intraparenchymal hemorrhages were excluded. Twelve patients experienced an unexpected aneurysmal subarachnoid hemorrhage complication after PED treatment, an incidence of $3.1 \%$ in our center $3.0 \%$ for PED with coils and $3.3 \%$ for PED alone). Two of these were excluded because of inadequate image quality for the computational simulation, so 10 patients with 10 PEDs were ultimately included in this study in the postoperative rupture (POR) group. For each case in the POR group, we enrolled 2 matched controls who did not have postoperative rupture and were analyzed as the postoperative unruptured (POU) group. The aneurysms in the control group should have had satisfactory angiographic results at follow-up, indicating a low probability of further rupture.

Aneurysms were graded on the degree of occlusion after PED placement as follows: complete occlusion (OG1), neck remnant (OG2), and residual sac filling (OG3). ${ }^{13}$ A satisfactory angiographic result was defined as OG1, OG2, or progressive occlusion. The primary basis for the matched controls was treatment method: PED alone or PED-assisted coil embolization. Additional matching was performed in the following order: aneurysm location, aneurysm size, patient sex, and age. To find the matched control cases, we retrospectively reviewed all unruptured aneurysms with PED placement seen in our clinic during the specified timeframe. We excluded the following patients: 1) those for whom the treatment method, location, and size of aneurysm could not be matched; 2) those without angiographic follow-up or 3D images with sufficient resolution for computational simulation; and 3) those having previous endovascular or surgical treatment of the aneurysm.

Twenty matched patients were included as the POU group. The patient characteristics of both groups are summarized (On-line Table 1). Clinical and morphologic factors for all 30 patients (age, sex, smoking history, hypertension, symptomatic aneurysm, aneurysm size, aneurysm neck, size ratio, and aspect ratio) were collected and calculated. The treatment method, coil packing density, and initial angiographic results were also collected.
For the Pipeline Embolization Device, the patients were treated with aspirin, $100 \mathrm{mg}$, and clopidogrel, $75 \mathrm{mg}$, for a minimum of 5 days before the endovascular treatment. The antiplatelet sensitivity test was performed routinely, and a booster dose of $300 \mathrm{mg}$ of clopidogrel was used if the patient was not sensitive to clopidogrel. After we achieved groin access, all patients received an initial heparin bolus administration (70-100 UI/kg) maintaining the activated clotting time between 250 and 300 seconds during the procedure. Dual-antiplatelet therapy (aspirin, $100 \mathrm{mg}$, and clopidogrel, $75 \mathrm{mg}$ ) will be maintained for 3 months after the procedure; then, aspirin monotherapy will be followed indefinitely.

\section{Computational Modeling and Hemodynamic Simulations}

Patient-specific 3D digital subtraction angiography data of all aneurysms were obtained and imported into Geomagic Studio, Version 12.0 software (Geomagic, Research Triangle Park, North Carolina). Images were displayed, segmented, and smoothed, and the surface geometries were saved as a Standard Tessellation Language format. We used a novel virtual stent placement technique ${ }^{14}$ and porous media method ${ }^{15}$ to simulate the in vivo stent and coil mass in the aneurysm dome region. The virtual stent placement consisted of 3 steps-1) preprocessing: the parent vessel was isolated from the aneurysm and trimmed down to the deployable region; 2) simplex mesh expansion: we obtained the centerline within the parent vessel and radial expansion to initialize a uniform small-diameter initial mesh (the expansion was stopped when the initialized simplex mesh was expanded inside the parent vessel with good apposition); and 3) postprocessing: the PED stent pattern was determined on the basis of the simplex mesh, and the wires were swept into the $3 \mathrm{D}$ structures using Computer Aided Design software (Creo Parametric 2.0; PTC, Needham, Massachusetts). ${ }^{14}$

The aneurysmal dome with coils was modeled as a porous medium described by Mitsos et al. ${ }^{15}$ The virtual stent was merged with the aneurysm geometry using ICEM CFD software (ANSYS, Canonsburg, Pennsylvania) to create $>1,000,000$ finite-volume tetrahedral elements. The maximum element size of the vessel was set at $0.2 \mathrm{~mm}$, and the element size of stents was approximately one-third the width of the strut of these stents. CFX 14.0 software (ANSYS) was used to simulate the hemodynamics of the aneurysm after meshing.

We treated blood as a Newtonian fluid. The blood vessel wall was assumed to be rigid with no-slip boundary conditions. The density was specified as $\rho=1060 \mathrm{~kg} / \mathrm{m}^{3}$, and the dynamic viscosity of blood was $\mu=0.004 \mathrm{~Pa} \times \mathrm{s}$. The governing equations underlying the calculation were based on the Navier-Stokes formula, with the assumption of homogeneous, laminar, incompressible blood flow. The inflow boundary condition was obtained using transcranial Doppler imaging as a representative pulsatile period velocity profile. The outlet pressure conditions at outlet arteries in our study were imposed to $P=0 \mathrm{~Pa}$. The flow waveforms were scaled to achieve a mean inlet wall shear stress (WSS) of 15 dyne/cm under pulsatile conditions. ${ }^{14}$ To confirm the stability, we selected the third of 3 cardiac cycle simulations as the output for the final analyses. 
Table 1: Univariate analysis results for hemodynamic parameters between POR and POU groups $^{\mathrm{a}}$

\begin{tabular}{lccc}
\multicolumn{1}{c}{ Parameters } & POR Group $(\boldsymbol{n}=10)$ & POU Group $(\boldsymbol{n}=\mathbf{2 0})$ & $\boldsymbol{P}$ Value \\
\hline Unstable flow (pre) (No.) (\%) & $3(30.0)$ & $10(50.0)$ & .297 \\
Unstable flow (post) (No.) (\%) & $6(60.0)$ & $4(20.0)$ & $.028^{\mathrm{b}}$ \\
Inflow jet (pre) (No.) (\%) & $8(80.0)$ & $15(75.0)$ & .760 \\
Inflow jet (post) (No.) (\%) & $2(20.0)$ & $9(45.0)$ & .180 \\
Flow complex (pre) (No.) (\%) & $4(40.0)$ & $9(45.0)$ & .439 \\
Flow complex (post) (No.) (\%) & $4(40.0)$ & $6(30.0)$ & .584 \\
Velocity (post) (m/s) & $0.03 \pm 0.04$ & $0.04 \pm 0.05$ & .286 \\
WSS (post) (Pa) & $1.42 \pm 2.55$ & $1.07 \pm 1.36$ & .792 \\
Pressure (post) (Pa) & $1487.31 \pm 799.68$ & $4127.68 \pm 5613.52$ & .930 \\
Velocity at neck (post) (m/s) & $0.30 \pm 0.33$ & $0.20 \pm 0.37$ & .567 \\
EL (post) (W/m ${ }^{3}$ ) & $906.32 \pm 707.56$ & $9871.75 \pm 22215.52$ & .628 \\
LSA (post) (mm $\left.{ }^{2}\right)$ & $0.56 \pm 0.32$ & $0.74 \pm 0.26$ & .147 \\
OSI (post) & $0.02 \pm 0.06$ & $0.00 \pm 0.00$ & .428 \\
TAWSS (post) (Pa) & $0.39 \pm 0.68$ & $0.23 \pm 0.29$ & .965 \\
RRT (post) (sec) & $1.46 \pm 2.54$ & $1.07 \pm 1.36$ & .725 \\
\hline
\end{tabular}

Note:-Pre indicates before; post, after.

${ }^{a}$ Continuous variables are expressed as mean \pm SD. Categoric variables are expressed as (No.) (\%).

${ }^{b} P<.05$. The Mann-Whitney $U$ test was used, with $P<.05$ considered statistically significant.
7) Relative residual time (RRT). RRT was defined as the inverse of the magnitude of the time-averaged WSS, which combined with WSS and oscillatory shear index. It quantifies the state of disturbed flow and reflects the duration of residence near the wall. ${ }^{21}$

To standardize the results, we used the reduction ratios of each of these parameters, and they were defined as (pretreatment parameter posttreatment parameter)/pretreatment parameter.

\section{Statistical Analysis}

We performed matched case-control analysis using conditional logistic regression. For categoric parameters, the $\chi^{2}$ test or the Fisher exact test was used to compare the differences

\section{Hemodynamic Analysis}

We then postprocessed and visualized the results of these simulations using the CFD-Post (ANSYS). The hemodynamic results at peak systole were carefully examined.

We calculated 7 hemodynamic variables:

1) Flow pattern. Flow complexity (simple/complex), flow stability (stable/unstable), and inflow jet were defined as Cebral et $\mathrm{al}^{16}$ reported. It was considered an improvement when the complex flow pattern turned to simple flow or the unstable flow pattern turned to stable flow. In flow complexity, a simple flow pattern consists of a single recirculation zone or vortex structure in the aneurysm, and a complex flow pattern contains $>1$ recirculation zone or vortex structure. With respect to flow stability, a stable flow pattern indicates that flow in an aneurysm is persistent during the cardiac cycle (does not move or change), and an unstable flow pattern is one in which the vortex structures are moved, created, or destroyed during the cardiac cycle.

2) WSS-related variables. The average WSS on the aneurysm sac was recorded. The time-averaged WSS (TAWSS) was calculated by integrating the WSS magnitude over the cardiac cycle and was normalized by the parent artery-averaged TAWSS in the same patient to allow comparison among different cases. ${ }^{17}$

3) Velocity. The average velocity in the aneurysm and the pressure on the aneurysm wall were recorded. The average flow velocity at the aneurysm neck plane was also recorded. The aneurysm neck plane was determined by a plane between the aneurysm sac and the parent artery.

4) Oscillatory shear index (OSI). The OSI was calculated using the method described by $\mathrm{He}$ and $\mathrm{Ku}^{18}$ and defined as the spatially averaged OSI on the aneurysm.

5) Low WSS area (LSA). LSA is the proportion of the low WSS area $(<10 \%$ of the mean vessel WSS at the parent artery) to the whole area of the aneurysm. ${ }^{19}$

6) Energy loss (EL). EL represents the expenditure of flow energy in the aneurysm region and was considered associated with aneurysm rupture. ${ }^{20}$ between the POR and POU groups. For continuous parameters, the Mann-Whitney $U$ test was used to compare the 2 groups. Statistical analyses were performed using statistical software (SPSS, Version 21.0; IBM, Armonk, New York). The level of statistical significance was established at $P<.05$.

\section{RESULTS}

\section{Case Matching, Clinical Factors, and Aneurysm Morphology}

In our study, 10 patients, each with an aneurysm, were included in POR group. After the primary matching and additional matching variables, each of the subjects with aneurysms was successfully matched with 2 control subjects with aneurysms. No PED malapposition was found in our included cases. For patients treated by PED and coils, the coiling and PED were performed in the same treatment session. The comparison of clinical and morphologic characteristics is shown in On-line Table 2. None of the clinical factors were statistically different between the 2 groups.

\section{Hemodynamic Analyses Associated with Postoperative Rupture}

We compared the pre- and postoperative flow patterns in each group and hemodynamic parameters between the 2 groups (Table 1).

\section{Qualitative Analyses}

Before treatment, the proportion of aneurysms with unstable flow patterns was $30 \%$ in the POR group and $50 \%$ in the POU group. The proportion of aneurysms with complex flow patterns was also lower in POR group than in the POU group, $40 \%$ versus $45 \%$, respectively. The proportion of aneurysms with an inflow jet was higher in the POR group ( $80 \%$ versus $75 \%$ ). There was no statistically significant difference between the 2 groups for these pretreatment measures.

After treatment, the proportion of the aneurysmal inflow jet decreased to $20.0 \%$ in the POR group and to $45.0 \%$ in the POU group. For complex flow patterns, there was no change in the 


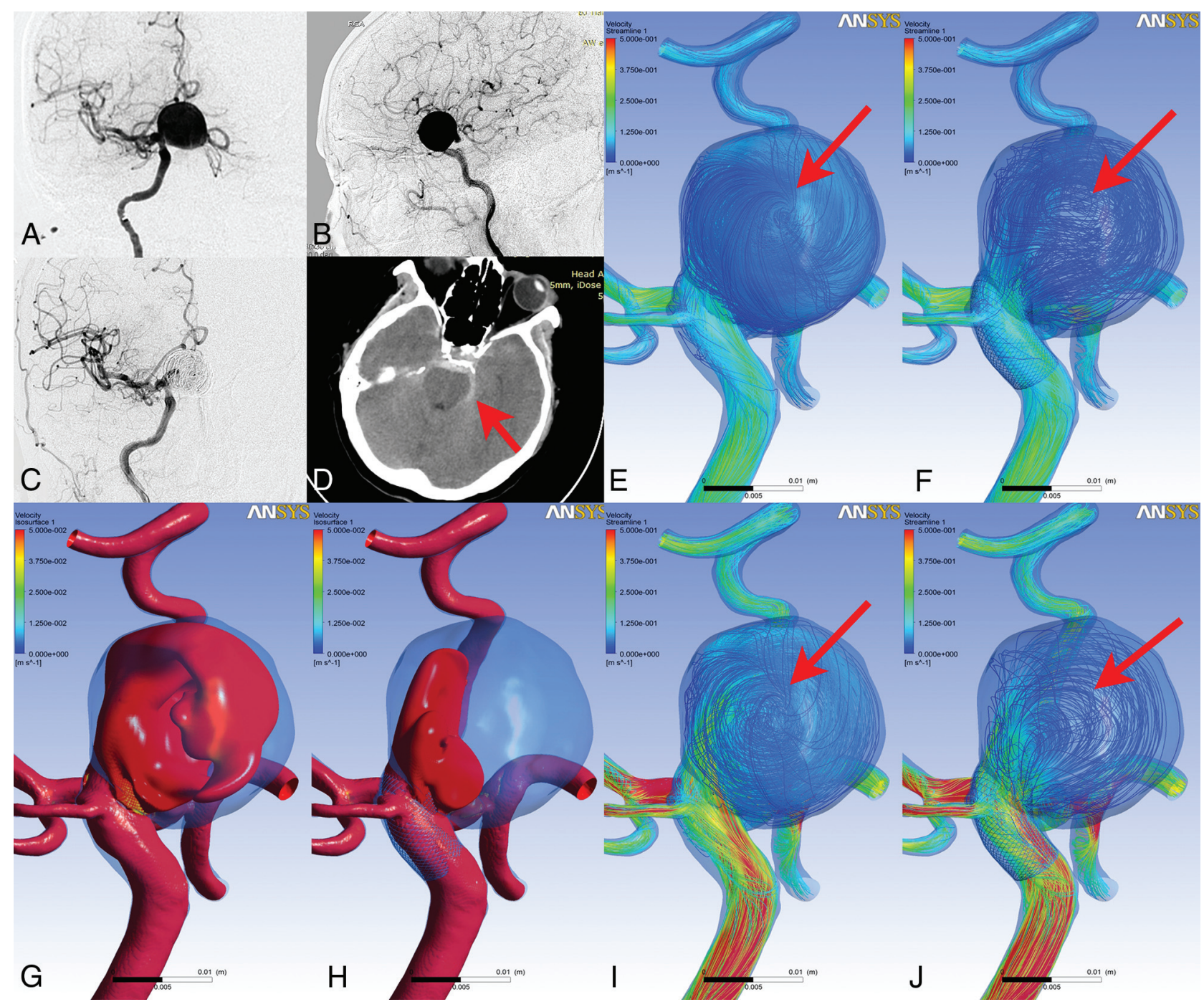

FIG 1. A female patient with a right internal carotid aneurysm was treated with PED-assisted coil embolization. Compared with the anteroposterior and lateral position of preoperative angiography $(A$ and $B)$, the aneurysm is embolized completely $(C)$. Unfortunately, the aneurysm ruptured 3 days postoperatively ( $D$, arrow). After CFD analysis, we found that an unstable flow pattern appeared after treatment. Compared with the streamlines at end diastole, the vortex structure in the preoperative streamlines ( $E$, arrow) is not changed at peak systole (I, arrow). However, after treatment, the vortex structure is moved at peak systole ( $F$ and $J$, arrows). Isovelocity surfaces show that the velocity in the aneurysm lumen decreased between the preoperative $(G)$ and postoperative $(H)$ periods.

POR group, while in the POU group, it decreased to 30\%. These differences showed no statistical significance. However, the proportion of aneurysms with unstable flow patterns did show a statistically significant difference between the 2 groups after treatment $(P=.028)$. The proportion of unstable flow patterns in the POR group increased to $60.0 \%$, while the proportion decreased to $20.0 \%$ in the POU group (Table 1). Figs 1 and 2 show the stable and unstable flow patterns of 2 cases, including a delayed-ruptured aneurysm with PED-assisted coils and a successfully cured aneurysm with PED-assisted coils. Moreover, 2 cases, including a delayed-ruptured aneurysm and a successfully cured aneurysm with PED-assisted coils, were also shown (Online Figs 1 and 2).

\section{Quantitative Analyses}

For the hemodynamic parameters after PED treatment, the flow velocity in the aneurysms was lower in the POR group compared with the POU group. The pressure, EL, and LSA of aneurysms were also lower for the POR group, while the WSS on the aneurysms, the velocity at the neck plane, OSI, TAWSS, and RRT of the aneurysms were higher. However, these differences were not statistically significant (Table 1).

We also compared the reduction ratio of these hemodynamic parameters before and after treatment (Fig 3). By means of the Mann-Whitney test, the reduction ratio of EL showed a significant difference between 2 groups $(P=.02)$. EL was decreased after treatment in the POU group (reduction ratio $=22.73 \% \pm$ $53.59 \%$ ) and increased in the POR group (reduction ratio = $-158.81 \% \pm 183.95 \%)$. The OSI and pressure of the aneurysms increased after treatment, while other parameters decreased after treatment. The reduction of lumen velocity (Figs 1-3), TAWSS, velocity at the neck plane, and WSS was lower in the POR group, while the reduction ratio of RRT and LSA in the POR group was 


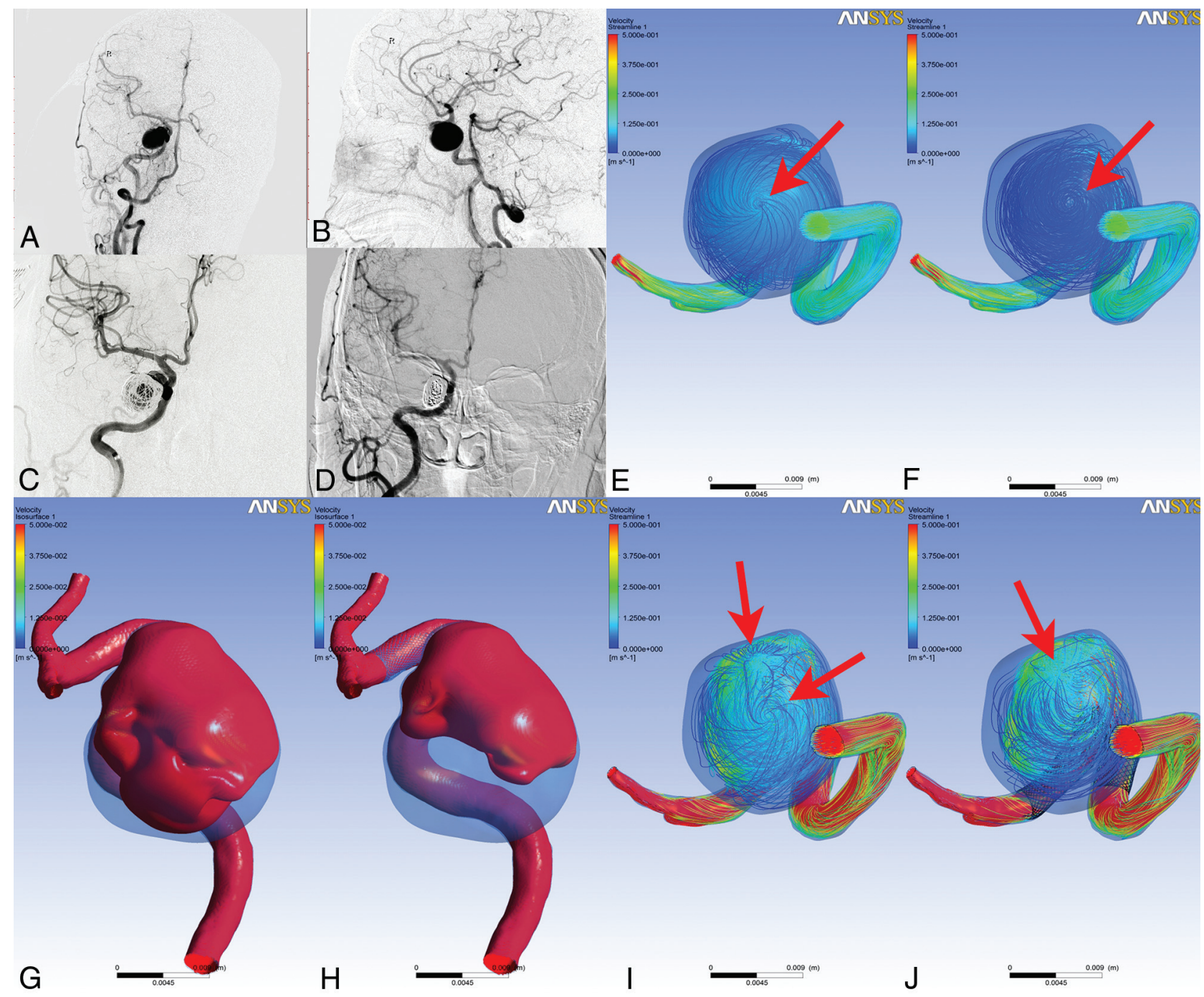

FIG 2. A female patient with a right internal carotid aneurysm treated with a PED-assisted coil embolization. Compared with the anteroposterior and lateral position of preoperative angiography $(A$ and $B)$, the aneurysm is embolized (C). At follow-up, the aneurysm is successfully embolized $(D)$. After CFD analysis, we found that the unstable flow pattern disappeared after treatment. Compared with the streamlines at end diastole, 1 vortex structure in the preoperative streamlines ( $E$, arrow) was found, and a created vortex appears at peak systole (I, arrow). However, after treatment, the amount of vortex structure is not changed at peak systole ( $F$ and $)$, arrows). Isovelocity surfaces show that the velocity in the aneurysm lumen decreased between the preoperative $(G)$ and postoperative $(H)$ periods.

higher. The difference in the reduction ratios between the 2 groups showed no statistical significance.

\section{DISCUSSION}

The mechanisms of postoperative rupture of IAs following flowdiversion treatment have been explored on the basis of clinical, morphologic, clinicopathologic, and hemodynamic characteristics. ${ }^{5,8,22,23}$ However, most studies were case reports or included many confounding factors, such as differing locations and sizes of aneurysms. In the present study, we adjusted for and matched these confounding factors with an aim of investigating the hemodynamic risk factors for postoperative rupture. We found that the unstable flow patterns were significantly increased in postoperative ruptured aneurysms. We also found higher EL in postoperative ruptured aneurysms after PED treatment, with the reduction ratio of EL showing a significant difference between the 2 groups $(P=0.02)$.

\section{Potential Delayed Ruptured Mechanism of Aneurysm after Flow Diverter}

Initial experience with flow-diverter technology was encouraging. However, some reports indicated that PED placement might lead to later aneurysm rupture, which raises concerns about the safety of this device. The incidence of subarachnoid hemorrhage after flow-diverter placement has been reported as high as $4.0 \%{ }^{5-9,22-24}$ Kulcsar et $\mathrm{al}^{8}$ analyzed risk factors of delayed-ruptured aneurysms before and after flow-diversion treatment to understand the mechanisms leading to delayed rupture during the healing process. They proposed 4 clinical risk factors for delayed rupture: large and giant aneurysms, symptomatic aneurysms, saccular aneurysms with an aspect ratio of $>1.6$, and inertia-driven inflow. However, this study mainly evaluated the clinical characteristics, and no hemodynamics were included. Treatment of large or giant aneurysms with flow diverters, which are considered at high risk of postoperative rupture, is still 


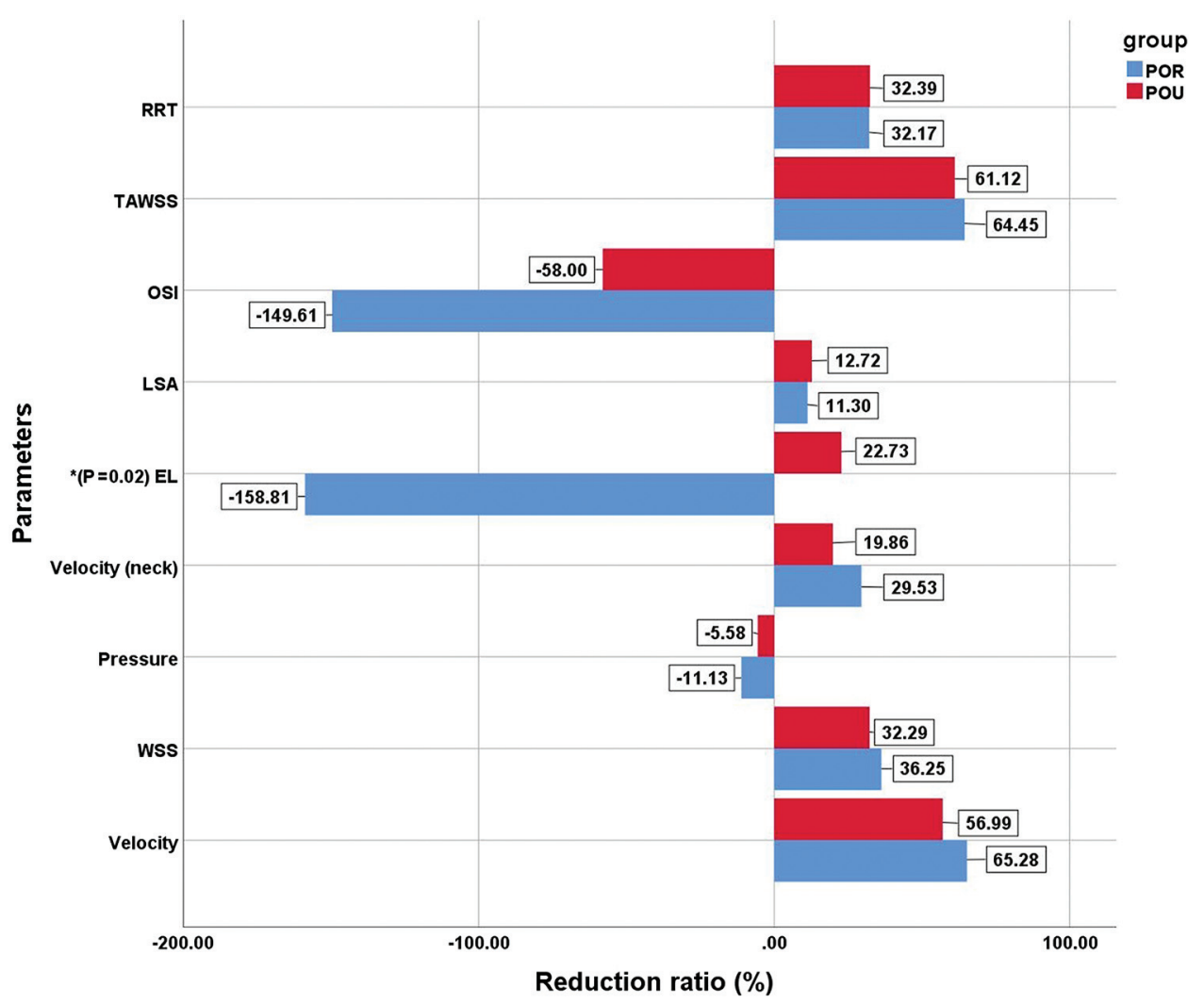

FIG 3. Comparisons of reduction ratios of hemodynamic parameters between the 2 groups. The asterisk indicates $P<.05$.

necessary, and the flow diverter is still a better choice than traditional stents. In our study, we primarily focused on hemodynamics because the flow diverter was designed to modify the aneurysmal flow. We strictly matched the control cases on the basis of clinical and morphologic factors and found that the hemodynamic risk factors indeed play a role in the postoperative rupture process.

A histologic study ${ }^{25}$ found that a fresh and organizing aneurysm lumen thrombus can induce degeneration of the aneurysm wall and was a risk factor for aneurysms predisposed to rupture. Inflammatory infiltration in the aneurysm wall was an independent risk factor associated with aneurysm rupture. However, it is not clear whether the mechanism of a postoperative ruptured aneurysm with PED treatment is related to intraluminal thrombus and inflammation. Similarly, several studies reported that unstable and nonorganized red thrombi can be the result of immediate stagnation of blood flow after flow-diverter placement. Red thrombi have a high content of proteolytic enzymes that can weaken the wall of the aneurysm. The subsequent thrombus organization within the aneurysm can result in inflammatory changes in the aneurysm wall, leading to delayed rupture of the aneurysm. ${ }^{7,10,11}$

Our hemodynamic results also supported this "thrombus and inflammatory" mechanism. We found that the flow velocity in aneurysms after PED placement was lower in the POR group and that the reduction ratio of aneurysmal velocity was higher, while the difference was not significant. Lower flow velocity in aneurysms after treatment and a higher reduction ratio of aneurysmal flow velocity could lead to rapid thrombosis in the aneurysm lumen and inflammatory infiltration of the aneurysm wall, which could trigger the aneurysm rupture. In addition, an unstable flow pattern increased after PED placement in the POR group, and such an unstable flow pattern might also result in rapid thrombosis in the aneurysm lumen. With the rapid intra-aneurysmal thrombus volume enlargement soon after flow-diverter placement, the aneurysm wall might tear from the mechanical stretching force on it.

In clinical practice, use of coils in conjunction with the PED is preferred to prevent delayed aneurysm rupture. ${ }^{26}$ Loose packing is usually performed in this circumstance, which is different from the dense packing in the conventional coil embolization. However, there is no guideline for the packing density in flow-diverter treatment, and there is also no solid evidence that coiling is the panacea. Shown in On-line Table 2 in our study, the difference in packing density was not significant between the 2 groups. The packing density may be too low to prevent delayed rupture in our data. Moreover, aneurysm rupture following flow-diverter therapy is a complex, multifactorial problem and needs to be further evaluated.

\section{The Hemodynamic Factors Related to Delayed Rupture of Aneurysms Treated with Flow Diverters}

Some computational fluid dynamics studies have tried to explain the mechanism of delayed rupture of aneurysms. Research has found that continued inflow and higher pressure in the aneurysm after flow-diverter stent placement might be potential mechanisms for delayed rupture. ${ }^{5,24}$ There are some limitations that might introduce bias to this conclusion. Hassan et $\mathrm{al}^{24}$ aimed to 
demonstrate possible adverse effects of deployment of flow-diverter stents. They found that a slow blood flow jet still exists inside the aneurysm at the end of the procedure, which might be related to delayed aneurysm rupture after treatment. However, the study was based on the flow characteristics of artificial saccular aneurysm models and did not relate the results to the clinical outcomes.

Cebral et $\mathrm{al}^{5}$ reported a computational fluid dynamics study in 3 patients who experienced postoperative rupture. They compared the hemodynamic factors with 4 successfully treated aneurysms and found that PED placement can increase intraaneurysmal pressure, which may potentially lead to rupture. However, the control cases were not strictly matched. Three of the 4 aneurysms were small- or medium-sized, so the flow pattern and hemodynamics may be different from those of giant aneurysms, and the small cases also have a low risk of postoperative rupture according to Kulcsar et al. ${ }^{8}$

In our study, we rigorously matched the control patients on the basis of clinical and morphologic factors. We found that the pressure in the aneurysm lumen increased after PED treatment, while the increasing ratio of pressure showed no significant difference between the POU and POR groups. Tateshima et $\mathrm{al}^{12}$ and Schneiders et $\mathrm{al}^{27}$ found similar results. These studies measured intrasaccular pressure before and after flow-diverter placement using pressure measurement wires and found no significant difference in intrasaccular pressures after flow-diverter placement.

Energy loss was introduced as a hemodynamic parameter, which is a more accurate estimate of the pressure variation and kinetic energy. ${ }^{28}$ Energy should be lost when the blood flow passes through the aneurysm. Part of this attenuated energy was quantified to EL as a hemodynamic parameter to judge whether an aneurysm had ruptured. Some studies have reported that EL was a useful parameter for the quantitative estimation of the rupture risk for IAs. Higher EL might be correlated to aneurysm rupture if the lost energy caused expansion of the aneurysm, leading to aneurysm rupture. ${ }^{20,28,29}$ However, until our study, there were no studies evaluating the role of EL in the process of postoperative rupture.

In this study, our results showed that EL was associated with postoperative aneurysm rupture. Higher energy loss after PED placement, then, might lead to delayed aneurysm rupture. As described by Takao et al, ${ }^{20} \mathrm{EL}$ is a hemodynamic parameter combining velocity and pressure. Our results showed that the flow velocity of the aneurysm lumen was decreased, while the pressure was increased. Although individually these parameters did not show a statistically significant difference between the 2 groups, the combined parameters, expressed as EL, did show a significant difference. Thus, EL might be a better hemodynamic parameter than pressure and velocity to predict aneurysmal delayed rupture after PED treatment.

\section{Limitations}

This study had some limitations. First, it was a retrospective analysis at a single center and lacked variety in the kind of flow-diverter device used; all cases were treated with a PED. Second, the study mainly aimed to investigate the hemodynamic factors and found that hemodynamic effects might play an important role in the causes of delayed rupture. However, the mechanism of aneurysm rupture following flow diversion is probably multifactorial and may include such things as antiplatelet regimen, thrombus formation, and inflammation. Last, several limitations of CFD hemodynamic analysis should be considered. Several assumptions, such as a rigid wall, laminar flow, and Newtonian blood, may affect the hemodynamic results, and the accuracy of the virtual stent placement technique still needs to be improved.

\section{CONCLUSIONS}

After PED treatment, an unstable flow pattern and higher energy loss compared with pretreatment might be the important hemodynamic factors related to delayed rupture of aneurysms. These analyses highlight the potential for CFD to play an important role in the clinical determination of POU IA risks.

\section{REFERENCES}

1. Walcott BP, Stapleton CJ, Choudhri O, et al. Flow diversion for the treatment of intracranial aneurysms. JAMA Neurol 2016;73:100208 CrossRef Medline

2. Kallmes DF, Brinjikji W, Cekirge S, et al. Safety and efficacy of the Pipeline Embolization Device for treatment of intracranial aneurysms: a pooled analysis of 3 large studies. J Neurosurg 2017; 127:775-80 CrossRef Medline

3. Becske T, Kallmes DF, Saatci I, et al. Pipeline for uncoilable or failed aneurysms: results from a multicenter clinical trial. Radiology 2013; 267:858-68 CrossRef Medline

4. Becske T, Brinjikji W, Potts MB, et al. Long-term clinical and angiographic outcomes following Pipeline embolization device treatment of complex internal carotid artery aneurysms: five-year results of the Pipeline for Uncoilable or Failed Aneurysms Trial. Neurosurgery 2017;80:40-48 CrossRef Medline

5. Cebral JR, Mut F, Raschi M, et al. Aneurysm rupture following treatment with flow-diverting stents: computational hemodynamics analysis of treatment. AJNR Am J Neuroradiol 2011;32:27-33 CrossRef Medline

6. Siddiqui AH, Kan P, Abla AA, et al. Complications after treatment with Pipeline embolization for giant distal intracranial aneurysms with or without coil embolization. Neurosurgery 2012;71:E509-13; discussion E513 CrossRef Medline

7. Chow M, McDougall C, O'Kelly C, et al. Delayed spontaneous rupture of a posterior inferior cerebellar artery aneurysm following treatment with flow diversion: a clinicopathologic study. AJNR Am J Neuroradiol 2012;33:E46-51 CrossRef Medline

8. Kulcsar Z, Houdart E, Bonafe A, et al. Intra-aneurysmal thrombosis as a possible cause of delayed aneurysm rupture after flow-diversion treatment. AJNR Am J Neuroradiol 2011;32:20-25 CrossRef Medline

9. Brinjikji W, Murad MH, Lanzino G, et al. Endovascular treatment of intracranial aneurysms with flow diverters: a meta-analysis. Stroke 2013;44:442-47 CrossRef Medline

10. Turowski B, Macht S, Kulcsar Z, et al. Early fatal hemorrhage after endovascular cerebral aneurysm treatment with a flow diverter (SILK-Stent): do we need to rethink our concepts? Neuroradiology 2011;53:37-41 CrossRef Medline

11. Hampton T, Walsh D, Tolias C, et al. Mural destabilization after aneurysm treatment with a flow-diverting device: a report of two cases. J Neurointerv Surg 2018;10:151-55 CrossRef Medline

12. Tateshima S, Jones JG, Mayor BF, et al. Aneurysm pressure measurement before and after placement of a Pipeline stent: feasibility study using a 0.014 inch pressure wire for coronary intervention. $J$ Neurointervent Surg 2016;8:603-07 CrossRef Medline 
13. Byrne JV, Beltechi R, Yarnold JA, et al. Early experience in the treatment of intra-cranial aneurysms by endovascular flow diversion: a multicentre prospective study. PLoS One 2010;5:e12492 CrossRef Medline

14. Wang C, Tian Z, Liu J, et al. Flow diverter effect of LVIS stent on cerebral aneurysm hemodynamics: a comparison with Enterprise stents and the Pipeline device. J Transl Med 2016;14:199 CrossRef Medline

15. Mitsos AP, Kakalis NM, Ventikos YP, et al. Haemodynamic simulation of aneurysm coiling in an anatomically accurate computational fluid dynamics model: technical note. Neuroradiology 2008;50:341-47 CrossRef Medline

16. Cebral JR, Mut F, Weir J, et al. Association of hemodynamic characteristics and cerebral aneurysm rupture. AJNR Am J Neuroradiol 2011;32:264-70 CrossRef Medline

17. Liu J, Fan J, Xiang J, et al. Hemodynamic characteristics of large unruptured internal carotid artery aneurysms prior to rupture: a case control study. J Neurointerv Surg 2016;8:367-72 CrossRef Medline

18. He $\mathrm{X}, \mathrm{Ku} \mathrm{DN}$. Pulsatile flow in the human left coronary artery bifurcation: average conditions. J Biomech Eng 1996;118:74-82 CrossRef Medline

19. Zhang Y, Jing L, Liu J, et al. Clinical, morphological, and hemodynamic independent characteristic factors for rupture of posterior communicating artery aneurysms. J Neurointerv Surg 2016;8:808-12 CrossRef Medline

20. Takao H, Murayama Y, Otsuka S, et al. Hemodynamic differences between unruptured and ruptured intracranial aneurysms during observation. Stroke 2012;43:1436-39 CrossRef Medline

21. Huang Q, Xu J, Cheng J, et al. Hemodynamic changes by flow diverters in rabbit aneurysm models: a computational fluid dynamic study based on micro-computed tomography reconstruction. Stroke 2013;44:1936-41 CrossRef Medline

22. Ikeda $\mathrm{H}$, Ishii A, Kikuchi $\mathrm{T}$, et al. Delayed aneurysm rupture due to residual blood flow at the inflow zone of the intracranial paraclinoid internal carotid aneurysm treated with the Pipeline Embolization Device: histopathological investigation. Interv Neuroradiol 2015;21:674-83 CrossRef Medline

23. Fox B, Humphries WE, Doss VT, et al. Rupture of giant vertebrobasilar aneurysm following flow diversion: mechanical stretch as a potential mechanism for early aneurysm rupture. $J$ Neurointerv Surg 2015;7:e37 CrossRef Medline

24. Hassan T, Ahmed YM, Hassan AA. The adverse effects of flow-diverter stent-like devices on the flow pattern of saccular intracranial aneurysm models: computational fluid dynamics study. Acta Neurochir (Wien) 2011;153:1633-40 CrossRef Medline

25. Frosen J, Piippo A, Paetau A, et al. Remodeling of saccular cerebral artery aneurysm wall is associated with rupture: histological analysis of $\mathbf{2 4}$ unruptured and $\mathbf{4 2}$ ruptured cases. Stroke 2004;35:2287-93 CrossRef Medline

26. Rouchaud A, Brinjikji W, Lanzino G, et al. Delayed hemorrhagic complications after flow diversion for intracranial aneurysms: a literature overview. Neuroradiology 2016;58:171-77 CrossRef Medline

27. Schneiders JJ, VanBavel E, Majoie CB, et al. A flow-diverting stent is not a pressure-diverting stent. AJNR Am J Neuroradiol 2013;34 E1-4 CrossRef Medline

28. Qian Y, Takao H, Umezu M, et al. Risk analysis of unruptured aneurysms using computational fluid dynamics technology: preliminary results. AJNR Am J Neuroradiol 2011;32:1948-55 CrossRef Medline

29. Liu J, Xiang J, Zhang Y, et al. Morphologic and hemodynamic analysis of paraclinoid aneurysms: ruptured versus unruptured. $J$ Neurointerv Surg 2014;6:658-63 CrossRef Medline 